\title{
Constraints on the location of a putative distant massive body in the Solar System from recent planetary data
}

\author{
Lorenzo Iorio ${ }^{1}$ \\ Ministero dell'Istruzione, dell'Università e della Ricerca (M.I.U.R.)-Istruzione. Fellow of \\ the Royal Astronomical Society (F.R.A.S.). International Institute for Theoretical Physics \\ and Advanced Mathematics Einstein-Galilei. Permanent address for correspondence: Viale \\ Unità di Italia 68, 70125, Bari (BA), Italy. \\ lorenzo.iorio@libero.it
}

Received __; accepted _ 


\begin{abstract}
We analytically work out the long-term variations caused on the motion of a planet orbiting a star by a very distant, pointlike massive object X. Apart from the semi-major axis $a$, all the other Keplerian osculating orbital elements experience long-term variations which are complicated functions of the orbital configurations of both the planet itself and of X. We infer constraints on the minimum distance $d_{\mathrm{X}}$ at which $\mathrm{X}$ may exist by comparing our prediction of the long-term variation of the longitude of the perihelion $\varpi$ to the latest empirical determinations of the corrections $\Delta \dot{\varpi}$ to the standard Newtonian/Einsteinian secular precessions of several solar system planets recently estimated by independent teams of astronomers. We obtain the following approximate lower bounds on $d_{\mathrm{X}}$ for the assumed masses of $\mathrm{X}$ quoted in brackets: $150-200$ au (Mars), $250-450$ au $\left(0.7 m_{\oplus}\right), 3500-4500$ au $\left(4 m_{\text {Jup }}\right)$.
\end{abstract}

Subject headings: Planets · Planet X · Solar System perturbations 


\section{Introduction}

Does not any other planet of the Sun exist in addition to those already known? Have not the remote peripheries of the solar system saved any further big surprises for us? It is a long time (Lowell 1915; Pickering 1928, 1931; Schuette 1949) that such questions-still well alive (Lykawka and Mukai 2008; Iorio 2010; Matese and Whitmire 2011; Fernández 2011) - are well rooted in the astronomical community, having often resurfaced in different

contexts and with changed forms;. For a recent, popular review, see Schilling (2009). Here we limit ourselves to recall that

- The hypothesis of a stellar-like companion object (Nemesis) orbiting the Sun was postulated for explaining terrestrial extinction periodicity (Raup and Sepkoski 1984), thought to be mediated by comet showers (Whitmire and Jackson 1984; Davis et al. 1984; Hills 1984; Hut 1984; Vandervoort and Sather 1993; Muller 2002). A recent critical review about the evidence for and against astronomical impacts on climate change and mass extinctions can be found in Bailer-Jones (2009); see Melott and Bambach (2010) for recent developments of the investigations on the role of Nemesis in such phenomena.

- Anomalies in the distribution of the aphelia and the orbital elements of the comets resident in the outer region of the Oort cloud led to postulating the existence of a Sun-bound Jovian mass body (Matese et al. 1999). Other studies on the interplay among a putative Planet X and comets are, e.g., Matese et al. (1986); Murray (1999); Horner and Evans (2002). For recent, quantitative investigations on such a Jupiter-type object, now named Tyche (Matese and Whitmire 2011) and not to be confused with Nemesis since it would not be able to induce comet storms, see Matese and Whitmire (2011). Another recent analysis can be found in Fernández (2011).

- The existence of an as yet undiscovered planet orbiting at trans-Plutonian distances was postulated (Maran et al. 1997; Collander-Brown et al.|2000; Brunini and Melita|2002; Melita et al. 2003, 2004; Matese et al. 2005; Gomes et al. 2006; Lykawka and Mukai 2008), with changeable fortune, to explain several features pertaining the dynamical history and the architecture of the Trans-Neptunian Belt. See Lykawka and Mukai (2008) for recent developments of such a scenario.

- A putative Planet X was also invoked (Gunn 1970; Rawlins 1970; Seidelmann 1971; van Flandern 1981; Anderson and van Flandern 1982; Anderson 1987; Seidelmann and Harrington 1988; Harrington 1988; Gomes and Ferraz-mello 1988; Gomes 1989; Powell 1989; van Flandern 1991) to explain certain seeming irregularities in the orbital motions of Uranus and Neptune which showed up in ancient data (Brunini 1992); such an issue was later settled by Standish (1993) with a re-analysis of the observations by including more recent radiometric points from the Voyager 2 
automatic spacecraft which allowed for a more accurate determination of the mass of Neptune. See Standish (1996) for issues related with the orbital residuals of Pluto, likely of non-dynamical origin.

- Harrison (1977) suggested that, as an explanation of the peculiar properties of certain pulsars with anomalously small period derivatives, the barycenter of the solar system is accelerated, possibly because of a hitherto undetected companion star of the Sun in a bound or open orbit. See also Cowling (1983) and Zakamska and Tremaine (2005) for further studies on such a topic.

- Iorio (2010) found that a distant, pointlike massive object in the outer regions of the solar system may explain the anomalous retrograde precession 1$]$ of the perihelion of Saturn preliminarily estimated (Pitjeva 2008) by analyzing some radiotechnical data points from the Cassini spacecraft exploring the Saturnian system.

Here by means of the expression "Planet X" we broadly denote a pointlike object, having a mass approximately as large as that of Mars or larger, up to typical values of brown dwarfs $\left(m_{\mathrm{X}} \approx 80 m_{\mathrm{Jup}}\right)$ or even red dwarfs $\left(m_{\mathrm{X}} \approx 0.5 \mathrm{M}_{\odot}\right)$ according to certain scenarios, located at large distances from the Sun, and for which no direct observational evidence, based on the detection of electromagnetic radiation of different wavelengths emitted or reflected by it in natural processes, is (yet?) available.

We also note that investigations on Planet X have direct connections with fundamental physics as well since it has been shown (Milgrom 2009; Blanchet and Novak 2011) that the action of a distant body located towards the Galactic center is dynamically equivalent to that of the External Field Effect (EFE) in the planetary regions of the solar system within the framework of the Modified Newtonian Dynamics (MOND). Moreover, Foot and Silagadze (2001) considered the possible existence of solar system's planets made of non-annihilating mirror matter, which is one of the candidates for the non-baryonic Dark Matter which is believed to constitute about $22 \%$ of the matter content of the Universe (Bertone et al. 2005). For other researches on the existence of mirror matter in the solar system, see Foot and Mitra (2003); a general overview on the mirror matter scenario and its observational implications can be found in, e.g., Foot (2002). For earlier studies on such a topic, see Blinnikov and Khlopov (1983); Khlopov et al. (1991); Khlopov (1999). Finally, the subtle perturbing effects due to a putative $\mathrm{X}$ may be important in realistically assessing the error budget in several high-precision tests of standard post-Newtonian gravity proposed or to be performed in the solar system arena (Iorio 2011).

In this paper, we use recent and accurate observational determinations of the motion of some planets of the solar system to put tighter constraints on the location of Planet $\mathrm{X}$

\footnotetext{
${ }^{1}$ Anyway, more recent data analyses (Pitjeva 2010) yield values for such an anomaly which are statistically compatible with zero. See Section 3 .
} 
with respect to those existing in literature. The plan of the paper is as follows. In Section 2 we analytically work out the effects that a distant body X would cause on the orbital motion of a closer and (relatively) fast-moving planet. More specifically, we adopt the Lagrange perturbative scheme to calculate, without simplifying approximations concerning the orbital configurations of both the planet and of $\mathrm{X}$, the long-term variations of all the Keplerian orbital elements of the perturbed planet. In Section 3 we turn to the latest observational determinations of the planetary motions. In particular, we compare our theoretical prediction for the long-term variation of the perihelion with the most recent determinations of the corrections to the standard precessions of the perihelia of the major bodies of the solar system obtained by independent teams of astronomers with the latest ephemerides. Our goal is to look at the minimum distance at which Planet X may exist as a function of its position in the sky. In Section 4 we review the past and future sky surveys and compare our bounds on the minimum distance of $\mathrm{X}$ to their obtained and expected results. Section 5 is devoted to the conclusions.

\section{Analytical calculation}

Let us consider a remote, point-like object $\mathrm{X}$ of mass $m_{\mathrm{X}}$ and located at distance $r_{\mathrm{X}}$ from a central body of mass $M$. Its action on a closer test particle orbiting $M$ at distance $r$ can be modelled as due to the following quadrupolar potential (Hogg et al. 1991), accurate up to terms of order $\mathcal{O}\left(r^{2} / r_{\mathrm{X}}^{2}\right)$,

$$
U_{\mathrm{X}}=\frac{\mathcal{K}_{\mathrm{X}}}{2}\left[r^{2}-3(\boldsymbol{r} \cdot \hat{\boldsymbol{l}})^{2}\right]
$$

where

$$
\mathcal{K}_{\mathrm{X}} \doteq \frac{G m_{\mathrm{X}}}{r_{\mathrm{X}}^{3}}
$$

is the tidal parameter of $\mathrm{X}$, while $\hat{\boldsymbol{l}}=\left\{l_{x}, l_{y}, l_{z}\right\}$ is a unit vector directed towards $\mathrm{X}$ determining its position in the sky. We purposely will not adopt any specific parameterization for it in order to make our results as general as possible. In eq. (11) $\boldsymbol{r}=\{x, y, z\}$ refers to the perturbed test particle.

In order to compute the effects of eq. (1) on the orbital motion of a test particle we shall use standard perturbative techniques. The use the eccentric anomaly $E$ (Murray and Dermott 1999) as fast variable of integration turns out to be more convenient to make the forthcoming integration more tractable. The average of eq. (11) over one orbital revolution of the perturbed test particle is

$$
\left\langle U_{\mathrm{X}}\right\rangle \doteq\left(\frac{n}{2 \pi}\right) \int_{0}^{P_{\mathrm{b}}} U_{\mathrm{X}} d t=\frac{\mathcal{K}_{\mathrm{X}} a^{2}}{32} \mathcal{U}(I, \Omega, \omega ; \hat{\boldsymbol{l}})
$$


with

$$
\begin{aligned}
\mathcal{U} & \doteq-\left(2+3 e^{2}\right)\left(-8+9 l_{x}^{2}+9 l_{y}^{2}+6 l_{z}^{2}\right)-120 e^{2} \sin 2 \omega\left(l_{x} \cos \Omega+l_{y} \sin \Omega\right)\left[l_{z} \sin I+\right. \\
& \left.+\cos I\left(l_{y} \cos \Omega-l_{x} \sin \Omega\right)\right]-15 e^{2} \cos 2 \omega\left[3\left(l_{x}^{2}-l_{y}^{2}\right) \cos 2 \Omega+2\left(l_{x}^{2}+l_{y}^{2}-2 l_{z}^{2}\right) \sin ^{2} I-\right. \\
& \left.-4 l_{z} \sin 2 I\left(l_{y} \cos \Omega-l_{x} \sin \Omega\right)+6 l_{x} l_{y} \sin 2 \Omega\right]-6\left(2+3 e^{2}\right)\left[\left(l_{x}^{2}-l_{y}^{2}\right) \cos 2 \Omega \sin ^{2} I+\right. \\
& \left.+2 l_{z} \sin 2 I\left(l_{y} \cos \Omega-l_{x} \sin \Omega\right)+2 l_{x} l_{y} \sin ^{2} I \sin 2 \Omega\right]-3 \cos 2 I\left\{\left(2+3 e^{2}\right)\left(l_{x}^{2}+l_{y}^{2}-2 l_{z}^{2}\right)+\right. \\
& \left.+5 e^{2} \cos 2 \omega\left[\left(l_{x}^{2}-l_{y}^{2}\right) \cos 2 \Omega+2 l_{x} l_{y} \sin 2 \Omega\right]\right\} .
\end{aligned}
$$

In eq. (3) and eq. (44) $a, e, I, \Omega, \omega$ are the semi-major axis, the eccentricity, the inclination of the test particle's orbit to the reference $\{X, Y\}$ plane, the longitude of the ascending node and the argument of pericenter, respectively; $n \doteq \sqrt{G M / a^{3}}$ is the unperturbed Keplerian mean motion connected to the unperturbed orbital period $P_{\mathrm{b}}$ by $n=2 \pi / P_{\mathrm{b}}$. Note that eq. (3) and eq. (44) are exact: neither approximations in $e$ nor in $I$ were used. In the integration $\hat{l}$ was kept fixed over one orbital revolution of the perturbed test particle, as it is reasonable given the assumed large distance of $\mathrm{X}$ with respect to it.

The standard Lagrange planetary equations (Bertotti et al. 2003), in which the disturbing function, i.e. $\left\langle U_{\mathrm{X}}\right\rangle$ in our case, appears, can be used to straightforwardly derive the long-term variations of all the standard six Keplerian osculating orbital elements of the perturbed particle. We also considered the longitude of the pericenter $\varpi \doteq \Omega+\omega$ since its secular precession is one of the parameters usually estimated by the astronomers when they fit the dynamical force models of their ephemerides to huge planetary data records (Pitjeva) 2005, 2008, 2010; Fienga et al. 2010; Fienga 2010).

From the Lagrange planetary equation for the semi-major axis (Bertotti et al. 2003), it can be noted that $a$ does not change since eq. (4) does not contain the mean anomaly $\mathcal{M}$. Instead, all the other Keplerian orbital elements experience non-vanishing long-term variations. They are

$$
\frac{d e}{d t}=\frac{15 \mathcal{K}_{\mathrm{X}} e \sqrt{1-e^{2}}}{16 n} \mathcal{E}(I, \Omega, \omega ; \hat{\boldsymbol{l}})
$$

with

$$
\begin{aligned}
\mathcal{E} & \doteq-8 l_{z} \cos 2 \omega \sin I\left(l_{x} \cos \Omega+l_{y} \sin \Omega\right)+4 \cos I \cos 2 \omega\left[-2 l_{x} l_{y} \cos 2 \Omega+\right. \\
& \left.+\left(l_{x}^{2}-l_{y}^{2}\right) \sin 2 \Omega\right]+\sin 2 \omega\left[\left(l_{x}^{2}-l_{y}^{2}\right)(3+\cos 2 I) \cos 2 \Omega+2\left(l_{x}^{2}+l_{y}^{2}-2 l_{z}^{2}\right) \sin ^{2} I-\right. \\
& \left.-4 l_{z} \sin 2 I\left(l_{y} \cos \Omega-l_{x} \sin \Omega\right)+2 l_{x} l_{y}(3+\cos 2 I) \sin 2 \Omega\right]
\end{aligned}
$$




$$
\frac{d I}{d t}=\frac{3 \mathcal{K}_{\mathrm{X}}}{4 \sqrt{1-e^{2}} n} \mathcal{I}(I, \Omega, \omega ; \hat{\boldsymbol{l}})
$$

in which

$$
\begin{aligned}
\mathcal{I} & \doteq\left[l_{z} \cos I+\sin I\left(-l_{y} \cos \Omega+l_{x} \sin \Omega\right)\right]\left\{5 e^{2} l_{z} \sin I \sin 2 \omega+\right. \\
& \left.+5 e^{2} \cos I \sin 2 \omega\left(l_{y} \cos \Omega-l_{x} \sin \Omega\right)+\left[2+e^{2}(3+5 \cos 2 \omega)\right]\left(l_{x} \cos \Omega+l_{y} \sin \Omega\right)\right\}
\end{aligned}
$$

$$
\frac{d \Omega}{d t}=-\frac{\mathcal{K}_{\mathrm{X}}}{4 n \sqrt{1-e^{2}}} \mathcal{N}(I, \Omega, \omega ; \hat{\boldsymbol{l}})
$$

with

$$
\begin{aligned}
\mathcal{N} & \doteq 3 \csc I\left[l_{z} \cos I+\sin I\left(-l_{y} \cos \Omega+l_{x} \sin \Omega\right)\right]\left\{-2 l_{z} \sin I+\right. \\
& +\cos I\left[-2+e^{2}(-3+5 \cos 2 \omega)\right]\left(l_{y} \cos \Omega-l_{x} \sin \Omega\right)+ \\
& \left.+e^{2}\left[l_{z}(-3+5 \cos 2 \omega) \sin I-5 \sin 2 \omega\left(l_{x} \cos \Omega+l_{y} \sin \Omega\right)\right]\right\}
\end{aligned}
$$

$$
\frac{d \omega}{d t}=-\frac{3 \mathcal{K}_{\mathrm{X}}}{16 n \sqrt{1-e^{2}}} \mathcal{P}(I, \Omega, \omega ; \hat{\boldsymbol{l}})
$$

with

$$
\begin{aligned}
\mathcal{P} & \doteq-\left(1-e^{2}\right)\left(-8+9 l_{x}^{2}+9 l_{y}^{2}+6 l_{z}^{2}\right)+\left(l_{x}^{2}+l_{y}^{2}-2 l_{z}^{2}\right)\left[-2-3 e^{2}-5 \cos 2 I+\right. \\
& \left.+5 \cos 2 \omega\left(-1+2 e^{2}+\cos 2 I\right)\right]+\cos 2 \Omega\left\{( l _ { x } ^ { 2 } - l _ { y } ^ { 2 } ) \left[-1+6 e^{2}+\right.\right. \\
& \left.\left.+5\left(2 e^{2}-3\right) \cos 2 \omega+10 \cos 2 I \sin ^{2} \omega\right]-20\left(2-e^{2}\right) l_{x} l_{y} \cos I \sin 2 \omega\right\}+ \\
& +\quad 2 l_{z} \cos \Omega\left\{2 l_{y} \cot I\left[\left(1-e^{2}\right)(-3+5 \cos 2 \omega)+10 \cos 2 I \sin ^{2} \omega\right]-\right. \\
& \left.-5 l_{x}\left[2-3 e^{2}-\left(2-e^{2}\right) \cos 2 I\right] \csc I \sin 2 \omega\right\}-l_{z} \csc I \sin \Omega\left\{4 l_{x} \cos I[(1-\right. \\
& \left.\left.-\quad e^{2}\right)(-3+5 \cos 2 \omega)+10 \cos 2 I \sin ^{2} \omega\right]+10 l_{y} \sin 2 \omega\left[2-3 e^{2}-\right. \\
& \left.\left.-\left(2-e^{2}\right) \cos 2 I\right]\right\}+2 \sin 2 \Omega\left[-5\left(3-2 e^{2}\right) l_{x} l_{y} \cos 2 \omega+l_{x} l_{y}\left(-1+6 e^{2}+\right.\right. \\
& \left.\left.+10 \cos 2 I \sin ^{2} \omega\right)+5\left(2-e^{2}\right)\left(l_{x}^{2}-l_{y}^{2}\right) \cos I \sin 2 \omega\right] .
\end{aligned}
$$

$$
\frac{d \varpi}{d t}=\frac{\mathcal{K}_{\mathrm{X}}}{128 n \sqrt{1-e^{2}}}[\mathcal{G}(I, \Omega, \omega ; \hat{\boldsymbol{l}})+\mathcal{H}(I, \Omega, \omega ; \hat{\boldsymbol{l}})]
$$


where

$$
\begin{aligned}
-\frac{\mathcal{G}}{24\left(1-e^{2}\right)} & \doteq 8-9 l_{x}^{2}-9 l_{y}^{2}-6 l_{z}^{2}-10\left(l_{x}^{2}+l_{y}^{2}-2 l_{z}^{2}\right) \cos 2 \omega \sin ^{2} I+ \\
& +8 l_{z} \cos \Omega \sin I\left[l_{y} \cos I(-3+5 \cos 2 \omega)-5 l_{x} \sin 2 \omega\right]+ \\
& +\cos 2 \Omega\left[-3\left(l_{x}^{2}-l_{y}^{2}\right)\left(5 \cos 2 \omega+2 \sin ^{2} I\right)-40 l_{x} l_{y} \cos I \sin 2 \omega\right]- \\
& -8 l_{z} \sin I\left[l_{x} \cos I(-3+5 \cos 2 \omega)+5 l_{y} \sin 2 \omega\right] \sin \Omega+ \\
& +2\left[-3 l_{x} l_{y}\left(5 \cos 2 \omega+2 \sin ^{2} I\right)+10\left(l_{x}^{2}-l_{y}^{2}\right) \cos I \sin 2 \omega\right] \sin 2 \Omega- \\
& -\cos 2 I\left\{3\left(l_{x}^{2}+l_{y}^{2}-2 l_{z}^{2}\right)+5 \cos 2 \omega\left[\left(l_{x}^{2}-l_{y}^{2}\right) \cos 2 \Omega+2 l_{x} l_{y} \sin 2 \Omega\right]\right\}
\end{aligned}
$$

and

$$
\begin{aligned}
\mathcal{H} & \doteq-96\left[l_{z} \cos I+\sin I\left(-l_{y} \cos \Omega+l_{x} \sin \Omega\right)\right]\left\{-2 l_{z} \sin I+\right. \\
& +\cos I\left[-2+e^{2}(-3+5 \cos 2 \omega)\right]\left(l_{y} \cos \Omega-l_{x} \sin \Omega\right)+ \\
& \left.+e^{2}\left[l_{z}(-3+5 \cos 2 \omega) \sin I-5 \sin 2 \omega\left(l_{x} \cos \Omega+l_{y} \sin \Omega\right)\right]\right\} \tan (I / 2) .
\end{aligned}
$$

with

$$
\frac{d \mathcal{M}}{d t}=-\frac{\mathcal{K}_{\mathrm{X}}}{16 n} \mathcal{A}(I, \Omega, \omega ; \hat{\boldsymbol{l}})
$$

$$
\begin{aligned}
\mathcal{A} & \doteq\left(7+3 e^{2}\right)\left(-8+9 l_{x}^{2}+9 l_{y}^{2}+6 l_{z}^{2}\right)+120\left(1+e^{2}\right) \sin 2 \omega\left(l_{x} \cos \Omega+\right. \\
& \left.+l_{y} \sin \Omega\right)\left[l_{z} \sin I+\cos I\left(l_{y} \cos \Omega-l_{x} \sin \Omega\right)\right]+15\left(1+e^{2}\right) \cos 2 \omega\left[3 \left(l_{x}^{2}-\right.\right. \\
& \left.-l_{y}^{2}\right) \cos 2 \Omega+2\left(l_{x}^{2}+l_{y}^{2}-2 l_{z}^{2}\right) \sin ^{2} I-4 l_{z} \sin 2 I\left(l_{y} \cos \Omega-l_{x} \sin \Omega\right)+ \\
& \left.+6 l_{x} l_{y} \sin 2 \Omega\right]+6\left(7+3 e^{2}\right)\left[\left(l_{x}^{2}-l_{y}^{2}\right) \cos 2 \Omega \sin ^{2} I+2 l_{z} \sin 2 I\left(l_{y} \cos \Omega-\right.\right. \\
& \left.\left.-l_{x} \sin \Omega\right)+2 l_{x} l_{y} \sin ^{2} I \sin 2 \Omega\right]+3 \cos 2 I\left\{\left(7+3 e^{2}\right)\left(l_{x}^{2}+l_{y}^{2}-2 l_{z}^{2}\right)+\right. \\
& \left.+5\left(1+e^{2}\right) \cos 2 \omega\left[\left(l_{x}^{2}-l_{y}^{2}\right) \cos 2 \Omega+2 l_{x} l_{y} \sin 2 \Omega\right]\right\} .
\end{aligned}
$$

It is not possible to simplify such formulas by $a$ - priori orienting one of the reference axes along $\hat{\boldsymbol{l}}$ because the position of $\mathrm{X}$ is not known. In the case of the solar system, the reference $\{X, Y\}$ plane is typically the mean ecliptic at the epoch J2000.0, with the $X$ axis directed towards the mean equinox at the same epoch. Our calculation holds for any orientation of the reference frame. 
Other calculations, performed in the framework of the lunisolar perturbations on artificial planetary satellites, exist in literature (Kozai 1959; Cook 1962; Gurfil et al. 2007). They differ from ours in the choice of the orbital elements investigated, the calculational techniques adopted, and the representation of the unit vector of the disturbing body. A straightforward comparison with our results is, thus, not possible. Kozai (1959), by using a specific parameterization for the geocentric lunar unit vector $\hat{\boldsymbol{l}}$ in terms of some orbital elements of it, adopted the Lagrange planetary equations for $a, e, I, \Omega, \omega$. Anyway, he did not average the disturbing function over one orbital revolution of the satellite, retaining only the terms in which its mean longitude does not appear. Moreover, Kozai (1959) did not release explicit expressions for the variations of $e, I$ at all, while those for $\Omega$ and $\omega$ are presumably incomplete and difficult to evaluate since it is unclear how they were obtained. Cook (1962) used the more cumbersome Gauss2 equations for the variations of the osculating orbital elements to compute the long-term variations of $a, e, I, \Omega, \omega+\Omega \cos I$ by means of the true anomaly $f$ as fast variable of integration. Also Cook (1962) used a particular representation of the geocentric $\hat{\boldsymbol{l}}$, expressed in terms of its Keplerian orbital elements. Gurfil et al. (2007), working in the framework of a perturbed Martian satellite, employed the satellite's mean anomaly with the Lagrange planetary equations in the integration over one orbital revolution for $\dot{a}, \dot{e}, \dot{I}, \dot{\Omega}, \dot{\omega}$. They also adopted two specific parameterizations for $\hat{\boldsymbol{l}}$ related to different choices for the reference $\{X, Y\}$ plane.

Our results can be used not only in view of the fact that estimated corrections $\Delta \dot{\varpi}$ of the standard Newtonian/Einsteinian precessions of the longitudes of the perihelia are nowadays available for all the eight planets and Pluto (Pitjeva 2010; Fienga et al. 2010; Fienga 2010), but also because it seems that also the nodes will receive their due attention by the astronomers in the near future (Fienga 2010). The first determination of the corrections to their standard secular precession for the first six planets can be found in Fienga et al. (2011): we propose to use them in further analyses. Moreover, eq. (5)-eq. (16) can also be useful in severa $]^{3}$ exoplanetary scenarios. Indeed, in many cases the discovered extrasolar planets have distant companions (Howard et al. 2010), or their existence is postulated to explain certain observed features of the orbital motions (Collier Cameron et al. 2010).

\section{Confrontation with the observations}

In Table 1 we quote the latest determinations of the corrections $\Delta \dot{\varpi}$ to the standard Newtonian/Einsteinian secular precessions of the longitudes of the perihelia of all the major bodies of the solar system estimated by various authors with some of the most recent ephemerides. The effect of Planet $\mathrm{X}$ was not explicitly included in the dynamical force

\footnotetext{
${ }^{2}$ He dubbed them as Lagrange's planetary equations.

${ }^{3}$ See http://exoplanet.eu/ on the WEB.
} 
Table 1: Estimated corrections $\Delta \dot{\varpi}$, in milliarcseconds per century (mas cty ${ }^{-1}$ ), of the standard Newtonian/Einsteinian secular precessions of the longitudes of the perihelia $\varpi$ of the eight planets and Pluto determined with the EPM2008 (Pitjeva 2010), the INPOP08 (Fienga et al. 2010), and the INPOP10a (Fienga 2010) ephemerides. Concerning the values quoted in the third column from the left, they are those corresponding to the smallest uncertainties reported in (Fienga et al. 2010). Note the small uncertainty in the correction to the precession of the terrestrial perihelion, obtained by processing Jupiter VLBI data (Fienga et al. 2010).

\begin{tabular}{llll}
\hline Planet & $\Delta \dot{\varpi}($ Pitieva 2010 $)$ & $\Delta \dot{\varpi}($ Fienga et al. 2010) & $\Delta \dot{\varpi}$ (Fienga 2010) \\
\hline Mercury & $-4 \pm 5$ & $-10 \pm 30$ & $0.2 \pm 3$ \\
Venus & $24 \pm 33$ & $-4 \pm 6$ & - \\
Earth & $6 \pm 7$ & $0 \pm 0.016$ & - \\
Mars & $-7 \pm 7$ & $0 \pm 0.2$ & - \\
Jupiter & $67 \pm 93$ & $142 \pm 156$ & - \\
Saturn & $-10 \pm 15$ & $-10 \pm 8$ & $0 \pm 2$ \\
Uranus & $-3890 \pm 3900$ & $0 \pm 20000$ & - \\
Neptune & $-4440 \pm 5400$ & $0 \pm 20000$ & - \\
Pluto & $2840 \pm 4510$ & - & - \\
\hline
\end{tabular}

models fitted to the observations, so that, in principle, the extra-rates of the perihelia in Table 1 account for it. Here we shall compare our theoretical prediction of eq. (13) to the corrections of Table 1 in order to infer upper bounds on $\mathcal{K}_{\mathrm{X}}$, so to constrain the minimum distance $d_{\mathrm{X}}$ at which $\mathrm{X}$ may be located for different values of its mass $m_{\mathrm{X}}$.

By expressing the unit vector $\hat{\boldsymbol{l}}$ of $\mathrm{X}$ in terms of its ecliptic latitude $\beta_{\mathrm{X}}$ and longitude $\lambda_{\mathrm{X}}$ and by equating $\dot{\varpi}$ of eq. (13) to $\Delta \dot{\varpi}$ of Table 1 for each planet, it is possible to plot the minimum distance $d_{\mathrm{X}}$ of $\mathrm{X}$ as a function of $\beta_{\mathrm{X}}, \lambda_{\mathrm{X}}$ for given values of its putative mass $m_{\mathrm{X}}$. In Figure 1 we plot such bounding surfaces obtained from the accurate extra-precessions of the perihelia of the Earth, Mars and Saturn (Fienga et al. 2010; Fienga 2010) for $m_{\mathrm{X}}=4 m_{\text {Jup }}$, corresponding to Tyche (Matese and Whitmire 2011), $m_{\mathrm{X}}=0.7 m_{\oplus}$, considered in the scenario by Lykawka and Mukai (2008), and $m_{\mathrm{X}}=m_{\text {Mars }}$. It turns out that the bounds on $d_{\mathrm{X}}$ at high ecliptic latitudes are rather independent of $\lambda_{\mathrm{X}}$. For each of the perihelia there are a few sky locations, mainly close to the ecliptic, in which the dynamically inferred minimum distances of $\mathrm{X}$ are unrealistically small. Anyway, such positions differ from each other in such a way that a compensation occurs. Thus, a Tyche-like body cannot be located at less than about 3500 - 4500 au, an Earth-sized mass should not exist at less than about $250-450 \mathrm{au}$, while a rocky planet as big as Mars cannot be closer than approximately 150 - 200 au. The bounding surfaces by the Saturnian perihelion are generally smoother, with a milder excursion among the extremum 

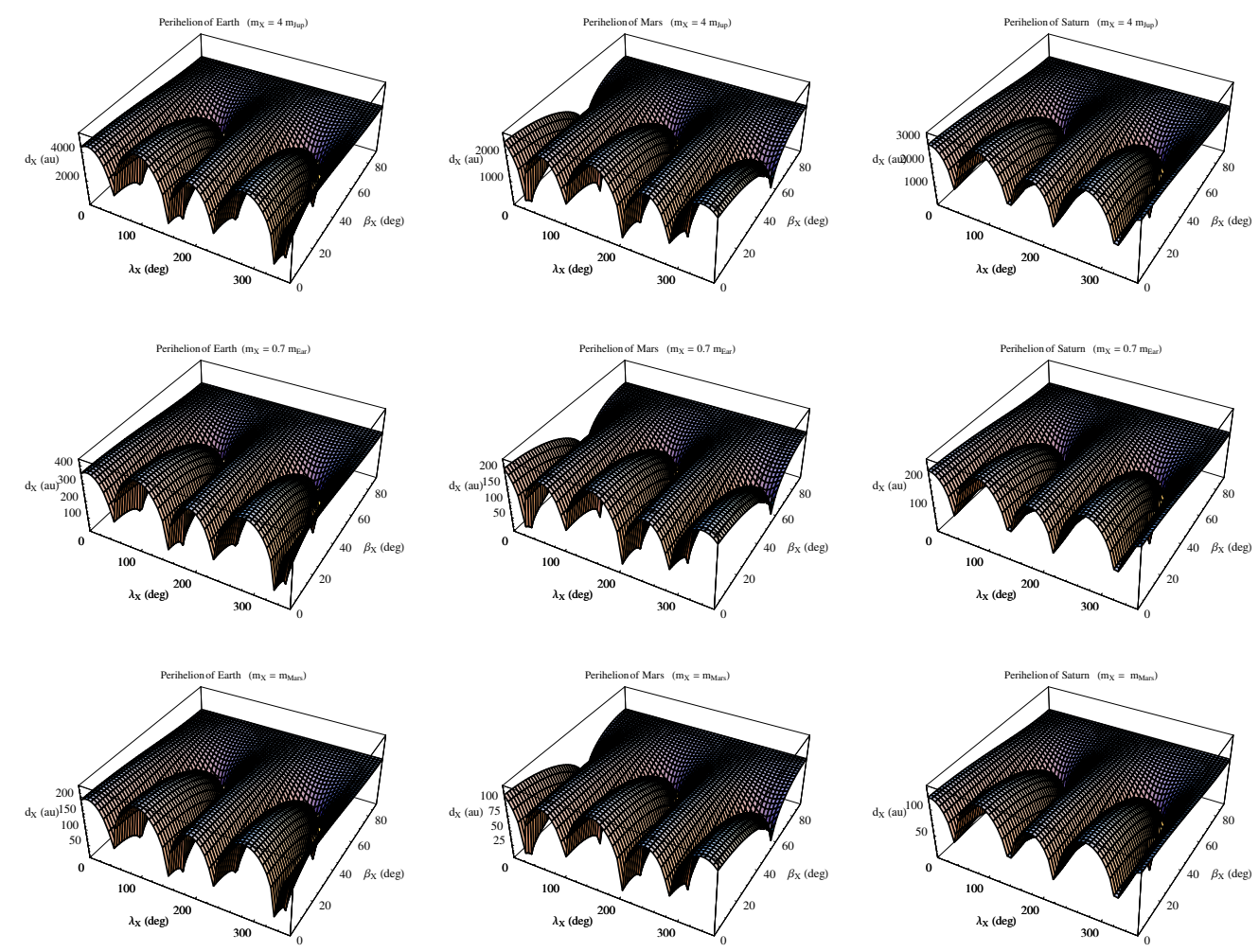

Fig. 1.- Constraints on the minimum distance $d_{\mathrm{X}}$, in au, for $m_{\mathrm{X}}=4 m_{\text {Jup }}$ (first row from the top), $m_{\mathrm{X}}=0.7 m_{\oplus}$ (second row from the top) and $m_{\mathrm{X}}=m_{\text {Mars }}$ (third row from the top) as a function of the ecliptic latitude $\beta_{\mathrm{X}}$ and longitude $\lambda_{\mathrm{X}}$ of $\mathrm{X}$. The perihelia of the Earth (left column), Mars (middle column) and Saturn (right column) were used according to $\Delta \dot{\varpi}_{\oplus}=0 \pm 0.016$ mas cty $^{-1}$ and $\Delta \dot{\varpi}_{\text {Mars }}=0 \pm 0.2$ mas cty $^{-1}$ (Fienga et al. 2010), and $\Delta \dot{\varpi}_{\text {Sat }}=0 \pm 2$ mas cty $^{-1}$ (Fienga 2010).

values. They are rather close to those determined by the perihelion of the Earth, whose extra-precession is 2 orders of magnitude more accurate than for Saturn (cfr. Table 11). Future improvements in the accuracy in determining the orbit of the ringed planet from continuous tracking of Cassini will straightforwardly yield more stringent constraints. It is possible to infer $d_{\mathrm{X}}$ also for values of $m_{\mathrm{X}}$ different from those used in Figure 1, dubbed $\bar{m}_{\mathrm{X}}$, by scaling the corresponding lower bounds $\bar{d}_{\mathrm{X}}$ by the multiplicative factor $\xi_{\mathrm{X}} \doteq\left(m_{\mathrm{X}} / \bar{m}_{\mathrm{X}}\right)^{1 / 3}$.

Our bounds are tighter than those obtained by Iorio 2009) with the correction to the precession of the perihelion of Mars estimated by Pitjeva (2005). 


\section{Possibilities of direct detections}

Concerning the ability of several performed, ongoing or planned wide area sky surveys to directly reveal a putative Planet $\mathrm{X}$ from its emitted or reflected electromagnetic radiation of different wavelengths, let us recall the following.

Limiting ourselves to optical wavelengths only, bodies as large as Jupiter or Neptune would have apparent visual magnitudes of about 20 and 23, respectively, at 1000 au, so that the largest telescopes may image them like just very dim dots (Fernández 2011). At 10000 au it would impossible to detect them with any optical telescope available today (Fernández 2011). Actually, our tightest constraints from the Earth's perihelion pose a jovian-sized object at not less than approximately 3000 - $4000 \mathrm{au}$; the minimum allowed distance for a Neptune-like mass would be of no more than about 1110 au. Since low-temperature bodies emit more efficiently in the infrared, surveys operating at such wavelengths are more suitable to look for Planet $\mathrm{X}$, especially those based in space because of the atmospheric extinction in the infrared.

All the wide area sky surveys performed so far mostly explored regions close to the ecliptic. The ecliptic, all-sky optical survey by (Tombaugh 1961) concluded that the minimum distance at which an Earth-sized body could be located is 81 au; similar findings were reached by the nearly ecliptic optical survey by Kowal (1989). Our dynamically inferred lower bounds are about 3-6 times more stringent. The Spacewatch optical survey (Larsen et al. 2007), conducted within 10 deg from the ecliptic, was sensitive to Mars-sized objects out to 300 au and Jupiter-sized planets out to $1200 \mathrm{au}$; its negative findings, especially with respect to the detection of a Jupiter-like body, are well explained by our analysis since its minimum distance dynamically inferred from the planetary perihelia is just more than 1000 au for those ecliptic latitudes. According to Matese and Whitmire (2011), Planet $X$ would have to be greater than $7-10 m_{\text {Jup }}$ and closer than $6000-25000$ au for a possible detection in the infrared, ground-based Two Micron All Sky Survey (2MASS) (Skrutskie et al. 2006). Our minimum distance for a body with $m_{\mathrm{X}}=7 m_{\text {Jup }}$ may be of the order of $1300 \mathrm{au}$ in a few locations near the ecliptic, and 4800 au for high latitudes. Matese and Whitmire (2011) note also that the negative search results of the InfraRed Astronomical Satellite (IRAS) survey (Neugebauer et al. 1984) suggest that an object with $m_{\mathrm{X}}=2-5 m_{\text {Jup }}$ must have a current minimum distance $d_{\mathrm{X}}=2000-10000$ au, respectively; our dynamically inferred bounds for the minimum allowed distance of such a $\mathrm{X}$ are in the range $\approx 3000-4500$ au for most of the positions in the sky. The all-sky synoptic Tycho-2 optical survey excluded the presence of a main-sequence star above the hydrogen-burning limit within $1 \mathrm{pc}=2.06 \times 10^{5}$ au $(\underline{\mathrm{H} g \mathrm{~g}} 2000)$.

\footnotetext{
${ }^{4}$ Certain rumors appeared in 1983 (O'Toole 1983) in mass-media about IRAS and Planet X, indirectly caused by Houck et al. (1984). Clarifications in specialistic literature, not spread by mass-media, appeared later (Houck et al. 1985; Soifer et al. 1987).
} 
Moving to ongoing or forthcoming projects, the all-sky synoptic survey by the Panoramic Survey Telescope and Rapid Response System (Pan-STARRS) (Jewitt 2003) would be able to reveal massive planets such as Neptune not beyond about 800 au, while a body with $m_{\mathrm{X}}=0.1 M_{\odot}$ would be undetectable for $r_{\mathrm{X}}>2000$ au. Conversely, our dynamically inferred lower bounds for such kind of bodies in the ecliptic are up to $\approx 1100$ au and $\approx 13400 \mathrm{au}$, respectively. A Jupiter-sized planet at 2000 au over the whole sky could, in principle, be detected by GAIA (Gaudi and Bloom 2005) with astrometric microlensing; actually, our lower limits for the admissible distance for such a body are globally more stringent. Also the putative existence of a X with $m_{\mathrm{X}}=3 m_{\mathrm{Jup}}$ at 20000 au could be put on the test (Gaudi and Bloom 2005). Possible planets in the outer regions ( $>1000$ $\mathrm{au}$ ) of the solar system may be revealed by the mesolensing technique (Di Stefano 2008). Another future all-sky survey which may be useful in detecting distant objects far from the ecliptic is the Large Synoptic Survey Telescope (LSST) (Stubbs et al. 2004). Babich et al. (2007) proposed to use observations of the Cosmic Microwave Background (CMB) to place constraints on the mass, distance, and size distribution of small objects in the Kuiper Belt and inner Oort Cloud. Maris and Burigana (2009) envisaged the possibility of using the data from the ongoing Planck mission to detect the thermal emission of pointlike objects of the solar system. The recently launched Wide-field Infrared Survey Explorer (WISE) (Wright et al. 2010) should be able to detect a Neptune-sized object out to 700 au, which is, in fact, a distance smaller than our dynamically inferred bounds on $d_{\mathrm{X}}$ for such a body. Instead, a Jupiter-mass object would be detectable out to 1 light year $=63239.7$ au, where it would still be within the Sun's zone of gravitational control. A larger object of 23 Jupiter masses would be visible at a distance of up to 7 to 10 light years (Lakdawalla 2009), i.e. $4.4-6.3 \times 10^{5}$ au. Actually, WISE has recently discovered its first ultra-cold brown dwarf, named WISEPC J045853.90+643451.9, at about 6 - 10 pc (Mainzer et al. 2011) corresponding to $1.2-2.0 \times 10^{6}$ au.

\section{Summary and conclusions}

We analytically computed the long-term variations of all the Keplerian osculating orbital elements of a test particle orbiting a primary and acted upon by a pointlike, distant object X. We assumed that it is located at a much larger distance $r_{\mathrm{X}}$ than the perturbed particle, so that the spatial position of $\mathrm{X}$ was kept fixed in the integration over one orbital revolution of the particle. With a perturbative, first-order calculation accurate up to terms of order $\mathcal{O}\left(r^{2} / r_{\mathrm{X}}^{2}\right)$ we found that all the Keplerian osculating orbital elements, apart from

its semi-major axis $a$, undergo long-term variations depending on the orbital geometries of both the perturbed and the perturbing objects in a complicated way.

We compared our analytical prediction for $\dot{\varpi}$ with the latest empirically determined corrections $\Delta \dot{\varpi}$ to the standard Newtonian/Einsteinian secular precessions of the perihelia of several solar system planets estimated by different teams of astronomers. We inferred 
constraints on the minimum distance $d_{\mathrm{X}}$ at which $\mathrm{X}$ may be located as a function of its ecliptic latitude $\beta_{\mathrm{X}}$ and longitude $\lambda_{\mathrm{X}}$ for different values of its mass $m_{\mathrm{X}}$. We adopted the masses of Mars, $0.7 m_{\oplus}$ and $4 m_{\text {Jup }}$. The use of the perihelia is effective in constraining $d_{\mathrm{X}}$ for most of the sky locations: the availability of $\Delta \dot{\varpi}$ for more than one planet allowed to mutually overlap the forbidden regions, thus further strengthening the lower bounds on $r_{\mathrm{X}}$ also in those positions of the sky in which the use of a single planet at a time would, instead, yield weaker constraints. The most stringent bounds came from the extra-rates of the perihelia of Saturn, Mars, and, especially, the Earth thanks to the inclusion of the Jupiter VLBI data. Future improvements for Saturn, due to continuous ranging to Cassini, should yield tighter constraints.

The minimum distance at which a remote body $\mathrm{X}$ with $70 \%$ of the mass of the Earth can exist is of the order of $250-450$ au. For other values of the mass of X, we have $150-200 \mathrm{au}$ (Mars), and 3500 - 4500 (4 $\mathrm{m}_{\mathrm{Jup}}$ ). Such lower bounds, which are independent of any speculation concerning the physical properties of X because they are solely based on its gravitational perturbations exerted on known planets, are tighter than those previously obtained by us in literature. Moreover, they are useful to better interpreting the results of past surveys aimed to directly detect X, and also to clarify what could realistically be expected from ongoing and future observational campaigns.

We intend to further extend our analysis in future by using the newly determined corrections to the standard secular precessions of the planetary perihelia and nodes as well by Fienga et al. (2011).

We remark that the corrections to the perihelion precessions used here were independently obtained by various astronomers without explicitly modeling the dynamical action of X itself. Thus, it might, in principle, have been somewhat "absorbed" and partially removed in the usual process of estimation of the solution's parameters like, e.g., the initial state vectors of the planets, especially if its magnitude was very small. To circumvent such an issue one should, actually, re-process the entire observational data set with modified dynamical force models explicitly including $\mathrm{X}$ itself, and solving for one or more dedicated parameters as well. Then, it would, e.g., be possible to look at the consequences of the inclusion of $\mathrm{X}$ on the values of the other usually estimated parameters. 


\section{REFERENCES}

Anderson, J.D., van Flandern, T.C.: Does the Sun have a Dark Companion? Newsweek 99, $83(1982)$

Anderson, J.D.: Still no Planet-X. Sky and Telescope 74, 237 (1987)

Babich, D., Blake, C., Steinhardt, C.: What Can the Cosmic Microwave Background Tell Us about the Outer Solar System? Astrophys. J., 669, 1406-1413 (2007)

Bailer-Jones, C.A.L.: The evidence for and against astronomical impacts on climate change and mass extinctions: a review. Int. J. of Astrobiol. 8, 213-219 (2009)

Bertone, G., Hooper, D., Silk, J.: Particle dark matter: evidence, candidates and constraints. Phys. Rept. 405, 279-390 (2005)

Bertotti, B., Farinella, P., Vokrouhlický, D.: Physics of the Solar System. Kluwer, Dordrecht (2003)

Blanchet, L., Novak, J.: External field effect of modified Newtonian dynamics in the Solar system. Mon. Not. Roy. Astron. Soc. 412, 2530-2542 (2011)

Blinnikov, S.I., Khlopov, M.Yu.: Possible Astronomical Effects of Mirror Particles. Sov. Astron. 27, 371-375 (1983)

Brunini, A.: On the unmodeled perturbations in the motion of Uranus. Celest. Mech. Dyn. Astron. 53, 129-143 (1992)

Brunini, A., Melita, M.D.: The Existence of a Planet beyond 50 AU and the Orbital Distribution of the Classical Edgeworth-Kuiper-Belt Objects. Icarus 160, 32-43 (2002)

Collander-Brown, S., Maran, M., Williams, I.P.: The effect on the Edgeworth-Kuiper Belt of a large distant tenth planet. Mon. Not. Roy. Astron. Soc. 318, 101-108 (2000)

Collier Cameron, A., Guenther, E., Smalley, B., McDonald, I., Hebb, L., Andersen, J., Augusteijn, Th., Barros, S.C.C., Brown, D.J.A., Cochran, W.D., Endl, M., Fossey, S.J., Hartmann, M., Maxted, P.F., Pollacco, D., Skillen, I., Telting, J., Waldmann, I.P., West, R.G.: Line-profile tomography of exoplanet transits - II. A gas-giant planet transiting a rapidly rotating A5 star. Mon. Not. Roy. Astron. Soc. 407, 507-514 (2010)

Cook, G.E.: Luni-Solar Perturbations of the Orbit of an Earth Satellite. Geophys. J. Int. 6, 271-291 (1962)

Cowling, S.A.: The pulsar $\dot{P} / P$ distribution and the postulated solar companion. Mon. Not. Roy. Astron. Soc. 204, 1237-1244 (1983) 
Davis, M., Hut, P., Muller, R.A.: Extinction of species by periodic comet showers. Nature 308, 715-717 (1984)

Di Stefano, R.: Mesolensing Explorations of Nearby Masses: From Planets to Black Holes. Astrophys. J. 684, 59-67 (2008)

Fernández, J.A.: On the Existence of a Distant Solar Companion and its Possible Effects on the Oort Cloud and the Observed Comet Population. Astrophys. J. 726, id. 33 (2011)

Fienga, A.: INPOP10a. Invited talk presented at Journées 2010 "Systèmes de référence spatio-temporels. New challenges for reference systems and numerical standards in astronomy", 20-22 September 2010, Paris (2010)

Fienga, A., Laskar, J., Kuchynka, P., Leponcin-Lafitte, C., Manche, H., Gastineau, M.: Gravity tests with INPOP planetary ephemerides. In: Klioner, S.A., Seidelmann, P.K., Soffel, M.H. (eds.) Relativity in Fundamental Astronomy: Dynamics, Reference Frames, and Data Analysis, Proceedings IAU Symposium No. 261, pp. 159-169. Cambridge University Press, Cambridge (2010)

Fienga, A., Laskar, J., Kuchynka, P., Manche, H., Desvignes, G., Gastineau, M., Cognard, I., Theureau, G.: The INPOP10a planetary ephemeris and its applications in fundamental physics. Celest. Mech. Dyn. Astron. 111, 363-385 (2011)

Foot, R., Silagadze, Z.K.: Do Mirror Planets Exist in Our Solar System? Acta Phys. Polon. B 32, 2271-2277 (2001)

Foot, R.: Shadowlands: Quest for Mirror Matter in the Universe. Universal Publishers, Parkland (2002)

Foot, R., Mitra, S.: Mirror matter in the solar system: new evidence for mirror matter from Eros. Astropart. Phys. 19, 739-753 (2003)

Gaudi, B.S., Bloom, J.S.: Astrometric Microlensing Constraints on a Massive Body in the Outer Solar System with Gaia. Astrophys. J. 635, 711-717 (2005)

Gomes, R.S.: On the problem of the search for Planet X based on its perturbation of the outer planets. Icarus 80, 334-343 (1989)

Gomes, R.S., Ferraz-Mello, S.: Would a Planet X explain the Discrepancies in the Motions of Uranus and Neptune? Anais Acad. Brasil. Ciências 60, 399-403 (1988)

Gomes, R.S., Matese, J.J., Lissauer, J.J.: A distant planetary-mass solar companion may have produced distant detached objects. Icarus 184, 589-601 (2006)

Gunn, E.J.: Another planet? New Sci. 48, 345 (1970) 
Gurfil P., Lainey V., Efroimsky M.: Long-term evolution of orbits about a precessing oblate planet: 3. A semianalytical and a purely numerical approach. Celest. Mech. Dyn. Astron. 99, 261-292 (2007)

Harrison, E.R.: Has the Sun a companion star? Nature 270, 324-326 (1977)

Harrington, R.S.: The location of Planet X. Astron J. 96, 1476-1478 (1988)

Hills, J. G.: Dynamical constraints on the mass and perihelion distance of Nemesis and the stability of its orbit. Nature 311, 636-638 (1984)

Høg, E., Fabricius, C., Makarov, V.V., Urban, S., Corbin, T., Wycoff, G., Bastian, U., Schwekendiek, P., Wicenec, A.: The Tycho-2 catalogue of the 2.5 million brightest stars. Astron. Astrophys. 355, L27-L30 (2000)

Hogg, D.W., Quinlan, G.D., Tremaine, S.: Dynamical limits on dark mass in the outer solar system. Astron J. 101, 2274-2286 (1991)

Horner, J., Evans, N.W.: Biases in cometary catalogues and Planet X. Mon. Not. Roy. Astron. Soc. 335, 641-654 (2002)

Houck, J.R., Soifer, B.T., Neugebauer, G., Beichman, C.A., Aumann, H.H., Clegg, P.E., Gillett, F.C., Habing, H.J., Hauser, M.G., Low, F.J., Miley, G., Rowan-Robinson, M., Walker, R.G.: Unidentified point sources in the IRAS minisurvey. Astrophys. J. 278, L63-L66 (1984)

Houck, J.R., Schneider, D.P., Danielson, G.E., Neugebauer, G., Soifer, B.T., Beichman, C.A., Lonsdale, C.J.: Unidentified IRAS sources - Ultrahigh-luminosity galaxies. Astrophys. J. 290, L5-L8 (1985)

Howard, A.W., Johnson, J.A.; Marcy, G.W., Fischer, D.A., Wright, J.T., Bernat, D., Henry, G.W., Peek, K.M.G., Isaacson, H., Apps, K., Endl, M., Cochran, W.D., Valenti, J.A., Anderson, J., Piskunov, N.E.: The California Planet Survey. I. Four New Giant Exoplanets. Astrophys. J. 721, 1467-1481 (2010)

Hut, P.: How stable is an astronomical clock that can trigger mass extinctions on Earth? Nature 311, 638-641 (1984)

Iorio, L.: Constraints on planet X/Nemesis from Solar System's inner dynamics. Mon. Not. Roy. Astron. Soc. 400, 346-353 (2009)

Iorio, L.: The Perihelion Precession of Saturn, Planet X/Nemesis and MOND. The Open Astron. J. 3, 1-6 (2010)

Iorio, L.: Effects of Standard and Modified Gravity on Interplanetary Ranges. Int. J. Mod. Phys. D 20, 181-232 (2011) 
Jewitt, D.: Project Pan-STARRS and the Outer Solar System. Earth, Moon, and Planets $92,465-476(2003)$

Khlopov, M.Yu., Beskin, G.M., Bochkarev, N.G., Pustilnik, L.A., Pustilnik, S.A.: Observational Physics of the Mirror World. Sov. Astron. 35, 21-29 (1991)

Khlopov, M.Yu.: Cosmoparticle physics. World Scientific, Singapore (1999)

Kowal, C.: A solar system survey. Icarus 77, 118-123 (1989)

Kozai, Y.: On the Effects of the Sun and the Moon upon the Motion of a Close Earth Satellite. Smithsonian Astronomical Observatory Special Report 22, part 2, 7-10 (1959)

Lakdawalla, E.: WISE Guys. In: The Planetary Society Blog, Aug. 27, 2009, http://www.planetary.org/blog/article/00002070/ (2009) Accessed 23 June 2011

Larsen, J.A., Roe, E.S., Albert, C.E., Descour, A.S., McMillan, R.S., Gleason, A.E., Jedicke, R., Block, M., Bressi, T.H., Cochran, K.C., Gehrels, T., Montani, J.L., Perry, M.L., Read, M.T., Scotti, J.V., Tubbiolo, A.F.: The Search for Distant Objects in the Solar System Using Spacewatch. Astron J. 133, 1247-1270 (2007)

Lowell, P.: Memoir on a trans-Neptunian planet. Mem. Lowell Obs. 1, No. 1, pp. 1-135 (1915)

Lykawka, P.S., Mukai, T.: An Outer Planet Beyond Pluto and the Origin of the Trans-Neptunian Belt Architecture. Astron J. 135, 1161-1200 (2008)

Mainzer, A., Cushing, M.C., Skrutskie, M., Gelino, C.R., Kirkpatrick, J.D., Jarrett, T., Masci, F., Marley Mark, S., Saumon, D., Wright, E., Beaton, R., Dietrich, M., Eisenhardt, P., Garnavich, P., Kuhn, O., Leisawitz, D., Marsh, K., McLean, I., Padgett, D., Rueff, K.: The First Ultra-cool Brown Dwarf Discovered by the Wide-field Infrared Survey Explorer. Astrophys. J. 726, id. 30 (2011)

Maran, M.D., Collander-Brown, S.J., Williams, I.P.: Limitations on the existence of a tenth planet. Planet. Space Sci. 45, 1037-1043 (1997)

Maris, M., Burigana, C.: On the Transits of Solar System Objects in the Forthcoming Planck Mission: Data Flagging and Coeval Multi-Frequency Observations. Earth, Moon, and Planets 105, 81-88 (2009)

Matese, J.J., Whitmire, D.P.: Planet X and the origins of the shower and steady state flux of short-period comets. Icarus 65, 37-50 (1986)

Matese, J.J., Whitman, P.G., Whitmire, D.P.: Cometary Evidence of a Massive Body in the Outer Oort Clouds. Icarus 141, 354-366 (1999) 
Matese, J.J., Whitmire, D.P., Lissauer, J.J.: A Widebinary Solar Companion as a Possible Origin of Sedna-like Objects. Earth, Moon, and Planets 97, 459-470 (2005)

Matese, J.J., Whitmire, D.P.: Persistent evidence of a jovian mass solar companion in the Oort cloud. Icarus 211, 926-938 (2011)

Melita, M.D., Williams, I.P.: Planet X and the Extended Scattered Disk. Earth, Moon, and Planets 92, 447-452 (2003)

Melita, M.D., Williams, I.P., Collander-Brown, S.J., Fitzsimmons, A.: The edge of the Kuiper belt: the Planet X scenario. Icarus 171, 516-524 (2004)

Melott, A.L., Bambach, R.K.: Nemesis reconsidered. Mon. Not. Roy. Astron. Soc. 407, L99-L102 (2010)

Milgrom, M.: MOND effects in the inner Solar system. Mon. Not. Roy. Astron. Soc. 399, 474-486 (2009)

Muller, R.: Measurement of the Lunar Impact Record for the Past 3.5 billion years, and Implications for the Nemesis Theory. Geol. Soc. of America Special Paper Vol. 356, Geol. Soc. of America, p. 659 (2002)

Murray, J.B.: Arguments for the presence of a distant large undiscovered Solar system planet. Mon. Not. Roy. Astron. Soc. 309, 31-34 (1999)

Murray, C.D., Dermott, S.F.: Solar System Dynamics. Cambridge University Press, Cambridge (1999)

Neugebauer, G., Habing, H.J., van Duinen, R., Aumann, H.H., Baud, B., Beichman, C.A., Beintema, D.A., Boggess, N., Clegg, P.E., de Jong, T., Emerson, J.P., Gautier, T.N., Gillett, F.C., Harris, S., Hauser, M.G., Houck, J.R., Jennings, R.E., Low, F.J., Marsden, P.L., Miley, G., Olnon, F.M., Pottasch, S.R., Raimond, E., Rowan-Robinson, M., Soifer, B.T., Walker, R.G., Wesselius, P.R., Young, E.: The Infrared Astronomical Satellite (IRAS) mission. Astrophys. J. 278, L1-L6 (1984)

O’Toole, T.: Mistery Heavily Body Discovered. The Washington Post, A1 (30th December 1983)

Pickering, W.H.: The next planet beyond Neptune. Popular Astron. 36, 143-165 (1928)

Pickering, W.H.: Planet P, its orbit, position and magnitude. Planets S and T. Popular Astron. 39, 385-398 (1931)

Pitjeva, E.V.: Relativistic Effects and Solar Oblateness from Radar Observations of Planets and Spacecraft. Astron. Lett. 31, 340-349 (2005) 
Pitjeva, E.V.: Ephemerides EPM2008: the updated model, constants, data. Paper presented at Journées "Systèmes de référence spatiotemporels" and X. Lohrmann-Kolloquium, 22-24 September 2008, Dresden, Germany (2008)

Pitjeva, E.V.: EPM ephemerides and relativity. In: Klioner, S.A., Seidelmann, P.K., Soffel, M.H., (eds.) Relativity in Fundamental Astronomy: Dynamics, Reference Frames, and Data Analysis, Proceedings IAU Symposium No. 261, pp. 170-178. Cambridge University Press, Cambridge (2010)

Powell, C.: A mathematical search for Planet X. J. Brit. Interplanet. Soc. 42, 327-333 (1989)

Raup, D.M., Sepkoski, J.J.: Periodicity of Extinctions in the Geologic Past. Proc. Natl. Acad. Sci. 81, 801-805 (1984)

Rawlins, D.: The Great Unexplained Residual in the Orbit of Neptune. Astron J. 75, 856-857 (1970)

Schilling, G.: The Hunt for Planet X. Springer, Berlin (2009)

Schuette, C.H.: Two new families of comets. Popular Astron. 57, 176-182 (1949)

Seidelmann, P.K.: A Dynamical Search for a Transplutonian Planet. Astron J. 76, 740-742 (1971)

Seidelmann, P.K., Harrington, R.S.: Planet X - The current status. Celest. Mech. Dyn. Astron. 43, 55-68 (1988)

Skrutskie, M.F., Cutri, R.M., Stiening, R., Weinberg, M.D., Schneider, S., Carpenter, J.M., Beichman, C., Capps, R., Chester, T., Elias, J., Huchra, J., Liebert, J., Lonsdale, C., Monet, D.G., Price, S., Seitzer, P., Jarrett, T., Kirkpatrick, J.D., Gizis, J.E., Howard, E., Evans, T., Fowler, J., Fullmer, L., Hurt, R., Light, R., Kopan, E. L., Marsh, K.A., McCallon, H.L., Tam, R., Van Dyk, S., Wheelock, S.: The Two Micron All Sky Survey (2MASS). Astron J. 131, 1163-1183 (2006)

Soifer, B.T., Neugebauer, G., Houck, J.R.: The IRAS view of the extragalactic sky. Ann. Rev. Astron. Astrophys. 25 187-230 (1987)

Standish, E.M.: Planet X - No dynamical evidence in the optical observations. Astron J. 105, 2000-2006 (1993)

Standish, E.M.: Pluto and Planets X. In: Rettig, T.W., Hahn, J.M. (eds.) Completing the Inventory of the Solar System, Astronomical Society of the Pacific Conference Proceedings, volume 107, pp. 163-170. (1996) 
Stubbs, C.W., Sweeney, D., Tyson, J.A.: An Overview of the Large Synoptic Survey Telescope (LSST) System. Bull. Am. Astron. Soc. 36, 1527 (2004)

Tombaugh, C.: The Trans-Neptunian Planet Search. In: Kuiper, G.P., Middlehurst, B.M. (eds.) Planets and Satellites, pp. 12-30. Univ. Chicago Press, Chicago (1961)

van Flandern, T.C., Pulkkinen, K.F., Santoro, E.J., Seidelmann, P.K., Harrington, R.S.: Perturbations of a Trans-Neptunian Planet. Bulletin of the American Astronomical Society, 13, 568 (1981)

van Flandern, T.C.: Planet-X. Sky and Telescope 82, 340-341 (1991)

Vandervoort, P.O., Sather, E.A.: On the Resonant Orbit of a Solar Companion Star in the Gravitational Field of the Galaxy. Icarus 105, 26-47 (1993)

Whitmire, D.P., Jackson, A.A.: Are periodic mass extinctions driven by a distant solar companion? Nature 308, 713-715 (1984)

Wright, E.L., Eisenhardt, P.R.M., Mainzer, A.K., Ressler, M.E., Cutri, R.M., Jarrett, T., Kirkpatrick, J.D.; Padgett, D., McMillan, R.S., Skrutskie, M., Stanford, S.A., Cohen, M., Walker, R.G., Mather, J.C., Leisawitz, D., Gautier, T.N., McLean, I., Benford, D., Lonsdale, C.J., Blain, A., Mendez, B., Irace, W.R., Duval, V., Liu, F., Royer, D., Heinrichsen, I., Howard, J., Shannon, M., Kendall, M., Walsh, A.L., Larsen, M., Cardon, J.G., Schick, S., Schwalm, M., Abid, M., Fabinsky, B., Naes, L., Tsai, C.-W.: The Wide-field Infrared Survey Explorer (WISE): Mission Description and Initial On-orbit Performance. Astron J. 140, 1868-1881 (2010)

Zakamska, N., Tremaine, S.: Constraints on the Acceleration of the Solar System from High-Precision Timing. Astron J. 130, 1939-1950 (2005)

This manuscript was prepared with the AAS LATEX macros v5.2. 